\title{
Avec ou sans famille proche à la vieillesse : une description du réseau de soutien informel des personnes âgées selon la présence du conjoint et des enfants WITH OR WITHOUT THE SUPPORT OF CLOSE FAMILY MEMBERS IN OLD AGE: A DESCRIPTION OF THE ELDERLY'S INFORMAL SUPPORT NETWORKS BASED ON THE PRESENCE OR ABSENCE OF A SPOUSE AND/OR CHILDREN
}

\section{Laurent Martel et Jacques Légaré}

Volume 30, numéro 1, printemps 2001

URI : https://id.erudit.org/iderudit/010300ar

DOI : https://doi.org/10.7202/010300ar

Aller au sommaire du numéro

Éditeur(s)

Association des démographes du Québec

ISSN

0380-1721 (imprimé)

1705-1495 (numérique)

Découvrir la revue

Citer cet article

Martel, L. \& Légaré, J. (2001). Avec ou sans famille proche à la vieillesse : une description du réseau de soutien informel des personnes âgées selon la présence du conjoint et des enfants. Cahiers québécois de démographie, 30(1), 89-114. https://doi.org/10.7202/010300ar

\section{Résumé de l'article}

Dans un contexte de plus en plus marqué par la désinstitutionnalisation des personnes âgées et le contrôle des dépenses reliées au domaine de la santé, nos sociétés mettent davantage à contribution le réseau social des individus en perte d'autonomie. Or ce réseau social est en pleine mutation. Avant de s'interroger sur sa disponibilité et sa composition futures, il y a lieu d'étudier dans quelles circonstances et comment, présentement, les personnes âgées utilisent leurs réseaux de soutien formels et informels. Comme les politiques de maintien à domicile varient d'une province canadienne à l'autre et que l'ISQ avait financé un suréchantillon de 700 personnes âgées pour l'Enquête sociale générale de Statistique Canada (1996), l'analyse porte sur la situation des personnes âgées du Québec recevant de l'aide parce qu'elles ont des problèmes de santé de longue durée (aînés vivant encore avec leur conjoint ; aînés ne vivant pas avec un conjoint mais ayant des enfants vivants ; et aînés sans conjoint ni enfants). Certes, toutes les personnes âgées n'ont pas besoin d'aide à cause de leur santé, mais les résultats soulèvent des questions pour l'avenir car la disponibilité du réseau informel sera nettement amoindrie. 
Cahiers québécois de démographie

Vol. 30, no 1, printemps 2001, p. 89-114.

\title{
Avec ou sans famille proche à la vieillesse : une description du réseau de soutien informel des personnes âgées selon la présence du conjoint et des enfants
}

\author{
Laurent MARTEL et Jacques LÉGARÉ *
}

\begin{abstract}
Dans un contexte de plus en plus marqué par la désinstitutionnalisation des personnes âgées et le contrôle des dépenses reliées au domaine de la santé, nos sociétés mettent davantage à contribution le réseau social des individus en perte d'autonomie. Or ce réseau social est en pleine mutation. Avant de s'interroger sur sa disponibilité et sa composition futures, il y a lieu d'étudier dans quelles circonstances et comment, présentement, les personnes âgées utilisent leurs réseaux de soutien formels et informels. Comme les politiques de maintien à domicile varient d'une province canadienne à l'autre et que l'ISQ avait financé un suréchantillon de 700 personnes âgées pour l'Enquète sociale générale de Statistique Canada (1996), l'analyse porte sur la situation des personnes agées du Québec recevant de l'aide parce qu'elles ont des problèmes de santé de longue durée (aînés vivant encore avec leur conjoint; aînés ne vivant pas avec un conjoint mais ayant des enfants vivants; et ainés sans conjoint ni enfants). Certes, toutes les personnes âgées n'ont pas besoin d'aide à cause de leur santé, mais les résultats soulèvent des questions pour l'avenir car la disponibilité du réseau informel sera nettement amoindrie. English abstract at the end of the article.
\end{abstract}

Dlus encore que le vieillissement progressif de la population, Pla gestion serrée des finances publiques a entrainé d'importantes réformes dans le domaine de la santé dans bon nombre de pays industrialisés. La désinstitutionnalisation des personnes âgées, le plus récent "virage ambulatoire " et les efforts qui sont faits pour limiter la durée des hospitalisations montrent clairement l'intention des décideurs publics de contenir les dé-

* Laurent Martel : Statistique Canada; Jacques Légaré : Département de démographie, Université de Montréal, et Statistique Canada. Les auteurs assument seuls l'entière responsabilité du contenu de cet article et des opinions qui y sont formulées, lesquelles ne représentent pas nécessairement les vues de Statistique Canada. Cet article est tiré d'un mémoire de maitrise soutenu au Département de démographie de 1'Université de Montréal en septembre 1995 (Martel, 1995). 
penses de santé en mettant davantage à contribution le réseau social de l'individu : sa famille proche (conjoint et enfants), sa famille élargie (frères et sœurs, oncles et tantes, neveux et nièces, cousins et cousines, etc.), ses amis et ses voisins.

Les personnes âgées forment un groupe particulièrement sensible à ces nouvelles politiques, car elles ont souvent besoin de soins durant de longues années en raison de leurs problèmes de santé. Arrivés dans le dernier quart de leur vie, les aînés, en particulier les femmes, doivent en outre composer avec la disparition progressive de certains êtres chers, frères, sœurs et surtout conjoint, qui les prive de précieux aidants potentiels. Les recherches menées à ce jour en gérontologie font systematiquement ressortir que le conjoint est la principale source de soins ${ }^{1}$ des ainés (Litwack, 1985; Antonucci et Akiyama, 1987; Stoller et Pugliesi, 1991; Thornton et WhiteMeans, 1993; Beggs et al., 1996). Certains auteurs ont même montré que son absence prédispose à vivre dans un établissement de santé (Cafferata, 1987; Pelletier, 1992; Trottier et al., 2000). Or, en 1996 au Québec, 386000 personnes âgées de 65 ans et plus (soit plus de $46 \%$ ) vivaient sans conjoint, et seules la plupart du temps ${ }^{2}$.

Pour les personnes seules, dont la plupart sont des femmes, mais aussi pour les personnes dont le conjoint vit toujours, les enfants, en particulier les filles, sont une importante source d'aide et de soins (Shanas et al., 1968; Chappell, 1991; Cantor, 1994; Stone et Rosenthal, 1997). Le nombre d'enfants mis au monde et surtout le nombre d'enfants encore vivants lorsque les parents atteignent l'âge de 65 ans prèsentent donc un intérêt certain dans l'étude du soutien social à la vieillesse (Wolf, 1994; Gee, 1995; Wu et Pollard, 1998; Giranda et al., 1999). Si de moins en moins de couples âgés ont aujourd'hui à déplorer la perte d'un enfant, certains sont demeurés inféconds. Au Québec, un peu plus de 124000 personnes âgées (soit environ $15 \%$ ) n'avaient plus d'enfants survivants en 1996; la plupart $(91770$, soit $74 \%)$ vivaient également sans conjoint.

Les personnes âgées sans conjoint et parfois sans enfants survivants peuvent avoir de la difficulté à conserver leur autonomie et à demeurer dans leur communauté. Lorsqu'elles sont en mesure de le faire, qui leur vient en aide? Sont-elles plus

Aide n'est pas synonyme de soins. Le second terme recouvre ici l'aide reliée à un problème de santé de longue durée (d'après Keating et al., 1999).

2 Presque trois personnes âgées sur quatre vivant sans leur conjoint vivent seules (Statistique Canada, Enquête sociale gẻnérale, cycle 11, 1996). 
nombreuses que les autres à compter sur les services formels d'aide à domicile?

Gérontologues, sociologues et démographes s'intéressent depuis plusieurs décennies à la taille et à la composition du réseau de soutien informel des personnes âgées les plus susceptibles de souffrir d'isolement: célibataires, veuves, divorcées, femmes sans enfants. Le réseau de soutien informel est composé des membres du réseau social qui aident la personne âgée à faire face à ses problèmes de santé de longue durée. L'état matrimonial et le mode de vie peuvent le modifier en influant sur la taille du réseau social et sur la disponibilité des aidants potentiels (Chappell, 1991; Connidis et McMullin, 1994; Barrett et Lynch, 1999). De nombreuses études montrent que les personnes âgées non mariées, en particulier les divorcées et les célibataires, et celles qui n'ont pas eu d'enfants sont plus nombreuses à compter des amis ou des voisins au sein de leur réseau de soutien informel et ont davantage recours au réseau formel que les personnes mariées (Chapman, 1989; Connidis et McMullin, 1994). Les veufs et surtout les veuves ont, plus que les autres, des liens étroits avec les membres de leur famille, surtout avec leurs frères et sœurs (Campbell et al., 1999).

C'est pourquoi nous nous intéressons au réseau de soutien informel des aînés. Nous en décrirons la taille et la composition, compte tenu du mode de vie, du nombre d'enfants survivants et de l'état matrimonial, en distinguant trois situations. Nous décrirons dans un premier temps le réseau de soutien informel de la majorité, soit les personnes âgées vivant encore avec leur conjoint (qu'elles vivent ou non avec d'autres personnes, apparentées à elles ou non); cette situation nous fournira un point de comparaison pour l'examen des autres situations. La deuxième situation est celle des personnes âgées qui ont perdu leur conjoint mais ont encore des enfants; nous tiendrons compte de l'état matrimonial, car la situation des veufs et veuves devrait différer de celle des divorcés(ées). La situation des personnes privées à la fois de conjoint et d'enfants fera l'objet de la dernière section.

\section{SOURCE DES DONNÉES}

Les données utilisées dans cet article proviennent du cycle 11 de l'Enquête sociale générale réalisée en 1996 par Statistique Canada, qui porte sur l'entraide et le soutien social. Deux des 
buts de cette enquête transversale sont de connaître la nature de l'aide donnée ou reçue et de comprendre la dynamique liant le réseau social d'une personne et l'aide qu'elle donne et (ou) reçoit. Le questionnaire a permis de recueillir des informations détaillées sur ce dernier point: préparation des repas, travaux ménagers, lessive et couture, entretien intérieur et extérieur de la maison, achats d'épicerie, déplacements, opérations bancaires et paiement de factures, soins personnels (bain, toilette, soin des mains, des pieds, des cheveux, hygiène dentaire, habillement), soutien moral ou émotif. Si ce type d'aide contribue sans aucun doute au bien-être des individus et surtout permet de combattre la solitude, il n'a cependant pas été retenu dans le présent article, centré sur les déterminants du maintien à domicile.

L'enquête permet également de connaitre la ou les raisons du besoin d'aide : aide reçue en raison d'un problème de santé, d'une situation difficile temporaire, du partage des tâches au sein du ménage, d'un manque de temps, etc. Seule l'aide reçue en raison d'un problème de santé de longue durée est prise en considération ici. Un aspect original de l'enquête réside dans le fait qu'elle renferme des informations - âge, sexe, lien avec le répondant, proximité géographique, fréquence des contacts sur chaque individu ayant fourni des soins (donc une aide liée à un problème de santé de longue durée) au répondant ou en ayant reçu de lui. Une liste exhaustive du réseau de soutien informel des personnes âgées est donc disponible dans l'enquête.

Les personnes âgées de 15 ans ou plus vivant dans des ménages privés au Canada constituaient la population cible de l'enquête (les pensionnaires d'établissements d'hébergement et les résidents du Yukon et des Territoires du Nord-Ouest étaient exclus de l'échantillon). La collecte des données a été réalisée au moyen d'entrevues téléphoniques assistées par ordinateur ${ }^{3}$. $\mathrm{Au}$ total, l'échantillon comptait 12756 répondants et le taux de réponse a été de $85,3 \%$. Dans la présente étude, seules les personnes âgées de 65 ans ou plus vivant au Québec ont été retenues, de sorte que la taille de l'échantillon utilisé est de 1744 répondants, représentant 832759 aînés vivant dans un ménage privé. Comme les politiques de maintien à domicile

Les ménages n'ayant pas le téléphone étaient donc systématiquement exclus (environ $2 \%$ des ménages canadiens selon Statistique Canada). Mais leurs caractéristiques ne diffèrent pas assez de celles du reste de la population visée pour influencer les estimations. Les pondérations de l'enquête tiennent compte de cet élément. 
varient d'une province canadienne à une autre, il paraît important de centrer l'analyse sur une seule province, ici le Québec. L'Institut de la statistique du Québec (ISQ) a financé un suréchantillonnage des personnes âgées de la province (700 répondants additionnels). N'ayant pas été conçu exclusivement pour l'étude de la population âgée, le cycle 11 de l'Enquête sociale générale ne fournit pas toujours de données fiables sur certains sous-groupes comme les personnes très âgées (80 ans et plus) ou les personnes sans conjoint ni enfants. Certains pourcentages ne peuvent donc ètre publiés en raison de coefficients de variation aléatoire trop élevés; ils sont remplacés, dans les tableaux, par le symbole "- ". Les résultats présentés reposent sur des données pondérées avec les poids de l'enquête.

\section{LES PERSONNES ÂGÉES VIVANT AVEC LEUR CONJOINT}

L'écart d'âge entre les époux et la mortalité différentielle entre les sexes sont deux éléments déterminants de la présence ou de l'absence du conjoint à la vieillesse. Le tableau 1 montre, sans causer de surprise, que les femmes àgées étaient, en 1996, plus souvent sans conjoint que les hommes âgés: trois hommes sur quatre et deux femmes sur cinq vivaient avec leur conjoint. Notons que la catégorie "personnes ãgées vivant avec leur conjoint " comprend les personnes vivant en union libre si le conjoint est présent dans le ménage. La surmortalité des époux, alliée au fait qu'ils sont généralement plus vieux que leurs épouses, contribue à la chute rapide de la proportion de femmes vivant avec conjoint : de 58\% à 65-69 ans, elle passe à $10 \%$ chez les 80 ans et plus. En comparaison, les deux tiers des hommes âgés de 80 ans ou plus bénéficient encore de la présence de leur conjointe, à un âge où les besoins d'aide et de soins sont particulièrement importants.

TABLEAU 1 - Pourcentage de personnes âgées vivant avec leur conjoint, Québec, $1996^{\text {a }}$

\begin{tabular}{lcccccc}
\hline & $\begin{array}{c}65-69 \\
\text { ans }\end{array}$ & $\begin{array}{c}70-74 \\
\text { ans }\end{array}$ & $\begin{array}{c}75-79 \\
\text { ans }\end{array}$ & $\begin{array}{c}80 \text { ans } \\
\text { et plus }\end{array}$ & Total & N \\
\hline Hommes & 74,7 & 77,8 & 73,7 & 67,9 & 74,4 & 447 \\
Femmes & 57,9 & 44,8 & 28,8 & 10,3 & 38,7 & 318 \\
Total & 65,7 & 59,0 & 46,5 & 29,7 & 53,6 & 765 \\
\hline
\end{tabular}

Source : Statistique Canada, Enquête sociale générale, cycle 11, 1996.

a. Italiques: la variabilité d'échantillonnage relative à cette estimation est élevée (trop dans certains cas pour que l'estimation soit publiée). 
Il apparaît d'ores et déjà qu'une inégalité importante existe entre hommes et femmes quant au soutien sur lequel ils peuvent compter à la vieillesse. Cependant, après un demi-siècle de hausse, l'écart entre l'espérance de vie des hommes et celle des femmes a commencé à décroître, signe que les écarts de mortalité entre les sexes diminuent (Nault, 1997). Il est donc probable que les futures cohortes d'aînés compteront une proportion de moins en moins élevée de veufs et de veuves, et que les conjoints pourront s'épauler plus longtemps lorsque viendront les problèmes de santé. Ce changement réduira-t-il le fardeau du système de santé ? Nous ferons quelques hypothèses à ce sujet. Mais avant de décrire le réseau social et le réseau de soutien informel des ainés, examinons quelques caractêristiques sociodémographiques susceptibles d'influencer leur capacité de trouver les soins dont ils ont besoin.

Il n'y a guère de différences entre les sexes pour la taille du ménage et la présence d'autres personnes au sein de ce dernier (tableau 2). On note toutefois certaines variations pour le revenu familial et l'état de santé, représenté ici par deux indicateurs, la santé perçue et l'indice de l'état de santé (IES). Ces variations peuvent être attribuables au fait que les deux populations étudiées constituent des univers différents, dont l'âge moyen, par exemple, n'est pas le même. L'écart d'âge entre les époux peut également expliquer le fait que plus de femmes que d'hommes déclarent des revenus inférieurs à 20000 dollars.

La taille et la composition du réseau social des hommes et des femmes âgées vivant encore avec leur conjoint présentent également quelques différences (tableau 3 ). Si le nombre moyen d'enfants survivants et le nombre moyen de frères et sours survivants sont comparables d'un sexe à l'autre, on note que les hommes ont dit se sentir proches d'un nombre plus élevé de personnes apparentées et d'amis. Ces différences sont à l'origine de la taille moyenne plus grande du réseau social des hommes $(17,8$ personnes, comparativement à 15,5 pour les femmes). Il est toutefois difficile de savoir si ces différences sont bien réelles puisque les questions de l'enquête font référence aux personnes apparentées et aux amis " proches " sans définir explicitement le mot "proche ", que les hommes ont pu interpréter autrement que les femmes. Quoi qu'il en soit, le nombre d'aidants potentiels parait très important chez les personnes âgées vivant encore avec leur conjoint. Théoriquement, elles pourraient donc combler sans difficulté leur besoin d'aide ou de soins si jamais ce dernier était incapable de le faire. 
TABLEAU 2 - Caractéristiques sociodémographiques des personnes âgées vivant avec leur conjoint, Québec, 1996 a

\begin{tabular}{lrrrr}
\hline & Hommes & Femmes & Total & $N$ \\
\hline Âge moyen (âge) & 72,3 & 70,8 & 71,7 & 765 \\
\hline Taille moyenne du ménage & 2,2 & 2,2 & 2,2 & 765 \\
\hline Mode de vie (\%) & & & & \\
$\quad$ Vit seulement avec conjoint & 83,1 & 84,0 & 83,5 & 639 \\
$\quad$ Vit avec conjoint et d'autres & 16,9 & 16,0 & 16,5 & 126 \\
$\quad$ Total & 100,0 & 100,0 & 100,0 & 765 \\
\hline Revenu familial (\%) & & & & \\
Moins de 10 000 \$ & - & - & - & -184 \\
10 000-19 999 \$ & 30,0 & 44,5 & 35,6 & 184 \\
20 000-29 999 \$ & 29,5 & 37,3 & 32,5 & 159 \\
30 000 \$ et plus & 39,3 & 18,1 & 31,0 & 140 \\
Total & 100,0 & 100,0 & 100,0 & 488 \\
\hline Etat de santé (\%) & & & & \\
Excellent, très bon & 47,0 & 37,5 & 43,1 & 313 \\
Bon & 30,5 & 38,5 & 33,8 & 276 \\
Moyen & 17,6 & 22,9 & 19,8 & 142 \\
Mauvais & 4,9 & - & 3,3 & 31 \\
Total & 100,0 & 100,0 & 100,0 & 762 \\
\hline IES b moyen (valeur) & 0,83 & 0,80 & 0,81 & 681 \\
\hline
\end{tabular}

Source : Statistique Canada, Enquête sociale générale, cycle 11, 1996.

a. Italiques et - : la variabilité d'échantillonnage relative à ces estimations est êlevée (trop dans certains cas pour que les estimations soient publiées).

b. Indice de l'êtat de santé. Indicateur mis au point par le Centre for Health Economics and Policy Analysis de I'Université McMaster (Canada), qui mesure l'état de santé fonctionnel global d'un individu à partir des huit attributs suivants : la vision, l'audition, l'élocution, la mobilité, la dextérité, la capacité cognitive, l'émotion ainsi que la douleur et les malaises. Cet indicateur situe les individus sur une échelle allant de 0 (la mort) à 1 (la parfaite santé). Pour une description complète de cet indicateur, voir Berthelot et al., 1993.

Environ un homme sur sept et une femme sur dix vivant avec son conjoint reçoit des soins (tableau 4). Cette proportion relativement faible est certainement liée à l'âge moyen présenté au tableau 2. La taille du réseau de soutien informel de ces personnes est de 1,6, nombre assez faible eu égard à la taille du réseau social. Si cette moyenne ne présente pas de différences selon le sexe, elle masque une distribution qui varie entre les hommes et les femmes. Les hommes déclarent un réseau social plus important que les femmes mais sont, assez paradoxalement, plus nombreux à recevoir des soins d'une 
TABLEAU 3 - Réseau social des personnes âgées vivant avec leur conjoint, Québec, 1996 a

\begin{tabular}{lccc}
\hline & Hommes & Femmes & Total \\
\hline \% sans enfants survivants & 6,2 & 8,7 & 7,2 \\
\hline Nombre moyen d'enfants survivants & 3,7 & 3,5 & 3,6 \\
$\begin{array}{l}\text { Nombre moyen de frères et scurs } \\
\text { survivants }\end{array}$ & 3,9 & 4,0 & 3,9 \\
$\begin{array}{l}\text { Nombre moyen de personnes apparentées } \\
\text { proches }\end{array}$ & 4,2 & 3,6 & 4,0 \\
Nombre moyen d'amis proches & 5,0 & 3,4 & 4,3 \\
\hline Taille moyenne du réseau social b & 17,8 & 15,5 & 16,8 \\
\hline
\end{tabular}

Source : Statistique Canada, Enquête sociale générale, cycle 11, 1996.

a. Italiques : la variabilité d'échantillonnage relative à ces estimations est élevée (trop dans certains cas pour que les estimations soient publiées).

b. Conjoint inclus.

seule personne, la plupart du temps leur femme (tableau 4). Une proportion beaucoup plus importante de femmes dẻclarent recevoir des soins de deux personnes seulement, la situation s'inversant de nouveau chez ceux qui reçoivent des soins de trois personnes ou plus.

TABLEAU 4-Réseau de soutien informel des personnes âgées vivant avec leur conjoint, Québec, $1996^{\text {a }}$

\begin{tabular}{lcrr}
\hline & Hommes & Femmes & Total \\
\hline \% de personnes âgées recevant des soins & 15,5 & 10,2 & 13,3 \\
IES moyen & 0,60 & 0,59 & 0,59 \\
Taille du ménage & 2,2 & 2,4 & 2,3 \\
\hline Nombre moyen de fournisseurs de soins & & & \\
$\quad 1$ & 69,5 & 56,1 & 65,1 \\
2 & - & 35,7 & 20,6 \\
$\quad 3$ et plus & 17,0 & - & 14,3 \\
\hline Moyenne & 1,6 & 1,5 & 1,6 \\
\hline Répartition (\%) selon que le fournisseur de & & & \\
soins : & & & \\
$\quad$ Vit dans le même mênage que l'aîné & 50,0 & 44,7 & 48,3 \\
$\quad$ Vit à l'extérieur du ménage de l'aîné & 50,0 & 55,3 & 51,7 \\
$\quad$ Total & 100,0 & 100,0 & 100,0 \\
\hline
\end{tabular}

Source : Statistique Canada, Enquête sociale générale, cycle 11, 1996.

a. Italiques et - : la variabilitè d'êchantillonnage relative à ces estimations est élevée (trop dans certains cas pour que les estimations soient publiées). 
TABLEAU 5 - Foumisseurs de soins des personnes âgées vivant avec leur conjoint, Québec, 1996 (\%) a

\begin{tabular}{lrrr}
\hline & Hommes & Femmes & Total \\
\hline Conjoint & 46,6 & 33,0 & 42,3 \\
Enfants & 14,4 & 22,4 & 16,9 \\
Membres de la famille b & 6,1 & 3,3 & 5,2 \\
Amis et voisins & 9,3 & - & 6,5 \\
Réseau de soutien formel c & 23,6 & 40,7 & 29,1 \\
Total & 100,0 & 100,0 & 100,0 \\
\hline
\end{tabular}

Source : Statistique Canada, Enquête sociale générale, cycle 11, 1996.

a. Italiques et - : la variabilité d'échantillonnage relative à ces estimations est élevée (trop dans certains cas pour que les estimations soient publiées).

b. Frères, sœurs, beaux-frères, belles-sœurs, gendres et brus ainsi que neveux et nièces.

c. Aide reçue d'un organisme non gouvernemental, d'un employé rémunéré ou d'un organisme gouvernemental.

Il est intéressant de constater que la moitié des soins fournis aux personnes âgées vivant avec leur conjoint sont donnés par des aidants extérieurs au ménage: le conjoint semble loin d'être l'unique source de soins. Les femmes, en particulier, vont chercher la plus grande partie de leurs soins hors du ménage, situation probablement liée d'abord à l'âge de leur époux mais peut-être aussi à une certaine division sexuelle du travail domestique. Il est en effet probable que les hommes de ces générations ne sont pas toujours en mesure de fournir les soins exigés par l'état de santé de leur conjointe en raison de leur inaptitude à accomplir certaines tâches, la préparation des repas par exemple. Pour cette raison, ces femmes vivant avec leur conjoint doivent trouver les soins dont elles ont besoin auprès d'autres aidants, situation moins fréquente chez les hommes.

Le tableau 5 , basé sur le nombre de dyades ${ }^{4}$, permet d'identifier les fournisseurs de soins des personnes âgées vivant avec leur conjoint. Au total, le conjoint fait partie d'une dyade sur deux dans le cas des hommes, et d'une sur trois dans le cas des femmes. Il est un peu surprenant que l'aide formelle soit la principale source de relations de soins des femmes. Cette situation ne se présente pas chez les hommes, qui comptent beaucoup plus fréquemment sur leurs amis et

4 Une dyade est une relation de soins entre le répondant et une autre personne. Comme un répondant peut avoir reçu des soins de plusieurs personnes de son réseau social, il est nécessaire de tenir compte du nombre total de relations de soins plutôt que du nombre de répondants. 
voisins. Les enfants fournissent également une part importante des soins, davantage pour les femmes que pour les hommes.

De toute évidence, les femmes âgées jouissant encore de la présence de leur conjoint ne sont pas toujours assurées de recevoir de lui les soins dont elles ont besoin. Plus âgé et plus susceptible d'être en moins bonne santé, peut-être moins habile dans certaines tâches domestiques, le conjoint n'est probablement pas toujours en mesure de fournir des soins. L'aide formelle et, dans une moindre mesure, les enfants, jouent par conséquent un rôle très important pour ces femmes (ces résultats confirment ceux de Shanas, 1979). On peut donc penser que la diminution attendue du nombre de veuves dans l'avenir n'allégera pas nécessairement la pression exercée sur le réseau formel de soins.

\section{LES PERSONNES ÂGÉES VIVANT SANS LEUR CONJOINT}

Presque une personne âgée sur deux ne vivant plus avec son conjoint pouvait compter, en 1996, sur au moins trois enfants survivants (tableau 6). Cette proportion relativement élevée est liée au fait que les générations âgées actuelles sont à l'origine, pour la plupart, du baby-boom canadien (Martel, 1998; Gee, 1995). Mais il faut remarquer qu'au sein des mêmes cohortes, une personne âgée sur quatre $(24 \%)$ n'avait pas d'enfant survivant. Si on peut s'attendre à ce que cette proportion décroisse encore un peu au cours des prochaines années, il apparait (tableau 6) que cette situation ne sera jamais négligeable au sein de la population âgée des prochaines décennies. Nous y reviendrons. Cette section porte sur les personnes âgées ne vivant plus avec leur conjoint et ayant encore des enfants au

TABLEAU 6 - Nombre d'enfants survivants des personnes âgées ne vivant pas avec leur conjoint, Québec, 1996 (\%) a

\begin{tabular}{lrrrrrr}
\hline & $\begin{array}{c}65-69 \\
\text { ans }\end{array}$ & $\begin{array}{c}70-74 \\
\text { ans }\end{array}$ & $\begin{array}{c}75-79 \\
\text { ans }\end{array}$ & $\begin{array}{c}80 \text { ans et } \\
\text { plus }\end{array}$ & Total & N \\
\hline 0 & 23,9 & 28,5 & 19,2 & 23,1 & 23,8 & 199 \\
1 & 9,3 & 11,1 & 11,3 & 15,9 & 12,0 & 100 \\
2 & 20,0 & 17,3 & 21,5 & 15,1 & 18,3 & 152 \\
3 et plus & 46,8 & 43,1 & 48,0 & 45,9 & 45,9 & 525 \\
Moyenne & 2,7 & 2,6 & 3,1 & 3,1 & 2,9 & 976 \\
\hline
\end{tabular}

Source : Statistique Canada, Enquête sociale générale, cycle 11, 1996.

a. Italiques : la variabilité d'échantillonnage relative à ces estimations est élevée (trop dans certains cas pour que les estimations soient publiées). 
sein de leur réseau social. L'analyse est faite selon l'état matrimonial, et non plus selon le sexe, puisque le veuvage ou la séparation et le divorce devraient influencer davantage la taille et la composition du réseau de soutien informel que le sexe (Morgan et March, 1992). On a d'ailleurs montré que les relations entre parents āgés et enfants adultes se dégradent suite au divorce des parents (Dykstra, 1997; Furstenberg, Hoffman et Shrestha, 1995; Uhlenberg, 1994; Pezzin et Schone, 1999).

\section{Les personnes âgées ne vivant pas avec leur conjoint et ayant des enfants}

Plus de quatre personnes âgées sur cinq ne vivant pas avec leur conjoint et ayant encore des enfants survivants étaient veuves en 1996; les autres étaient séparées ou divorcées (tableau 7). Très peu de personnes âgées de 75 ans et plus avaient vécu un divorce, car ce phénomène relativement récent touche davantage les cohortes plus jeunes. A 65-69 ans cependant, un individu sur trois vivant sans conjoint était divorcé, proportion non négligeable et très probablement appelée à s'accroitre (Martel et Carrière, 2001). D'une part, les adultes d'aujourd'hui, dont beaucoup sont nés durant le baby-boom, présentent des taux de divortialité importants. D'autre part, le divorce pourrait gagner en importance au-delà de cet âge, les aînés de demain ayant acquis ce comportement tôt dans leur cycle de vie.

TABLEAU 7 - État matrimonial des personnes ágées ne vivant pas avec leur conjoint et ayant des enfants survivants, Québec, $1996(\%)$ a

\begin{tabular}{lcccccc}
\hline & $\begin{array}{c}65-69 \\
\text { ans }\end{array}$ & $\begin{array}{c}70-74 \\
\text { ans }\end{array}$ & $\begin{array}{c}75-79 \\
\text { ans }\end{array}$ & $\begin{array}{c}80 \text { ans } \\
\text { et plus }\end{array}$ & Total & N \\
\hline Veufs(ves) & 63,0 & 82,1 & 96,6 & 97,4 & 84,8 & 640 \\
Séparés(ées), divorcés(ées) & 34,4 & 16,3 & - & - & 13,9 & 103 \\
Total & 97,4 & 98,4 & 96,6 & 97,4 & 98,7 & 743 \\
\hline
\end{tabular}

Source : Statistique Canada, Enquête sociale générale, cycle 11, 1996.

a. On compte 21 répondants mariès $(2,2 \%)$ et 10 célibataires $(1,2 \%)$ parmil la population ne vivant pas avec un conjoint et ayant au moins un enfant survivant lors de l'enquête. Ces nombres sont toutefois trop faibles pour permettre le calcul de statistiques fiables. Les répondants célibataires et les répondants mariés ont donc été omis de l'analyse. Pour cette raison, la ligne "Total " ne donne pas $100 \%$.

Italiques et - : la variabilité d'échantillonnage relative à ces estimations est êlevée (trop dans certains cas pour que les estimations soient publiées). 
De plus en plus d'études sont publiées sur les conséquences du divorce pour les personnes âgées. Beaucoup montrent que le réseau social des divorcés est plus limité que celui des veufs (Strain et Payne, 1992; De Jong Gierveld et Dykstra, 1997). Le divorce dégraderait les relations parents-enfants, en particulier entre les pères et leurs filles (Uhlenberg, 1994; Furstenberg et al., 1995; De Jong Gierveld et Dykstra, 1997; Pezzin et Schone, 1999). Il peut aussi avoir des conséquences pour la situation financière des individus, des femmes surtout (Keith, 1985; Uhlenberg, 1994). Nous nous attendons donc à constater d'importantes différences entre veufs et divorcés.

Le tableau 8 compare quelques caractéristiques sociodémographiques de ces deux groupes. La population des divorcés comprend une proportion à peu près égale d'hommes et de femmes, alors que les personnes frappées par le veuvage sont majoritairement des femmes. La population divorcée est également plus jeune en moyenne. Trois personnes sur quatre vivent seules, qu'elles soient veuves ou divorcées, même si elles ont des enfants survivants dans leur entourage. Cette proportion, très élevée, s'inscrit dans une tendance à la hausse, depuis trente ans, à la vie en solitaire au sein de la population âgée (Gee, 1995) et témoigne de la volonté actuelle des aînés de garder leur indépendance à l'égard de leurs proches. La disponibilité des aidants du réseau de soutien informel est donc d'autant plus importante pour permettre aux aînés de vivre autonomes dans leur communauté.

Une différence marquée entre les veufs et les divorcés apparaît cependant dans leur propension à vivre avec leurs enfants. La très grande majorité des veufs ne vivant pas seuls ( $22 \%$ des $25,8 \%$ vivent en fait avec un enfant. Chez les divorcés, un individu sur deux $(13,6 \%$ des $27,2 \%)$ vit avec d'autres personnes, qu'elles lui soient apparentées ou non. L'importance de l'écart donne à penser qu'au delà des diffêrences d'âge et d'état de santé observées entre ces groupes, les liens familiaux ont tendance à se dégrader après une rupture d'union. Les résultats obtenus ici rejoignent ceux de plusieurs études déjà citées. On peut se demander si le divorce a des effets similaires sur la taille du réseau social. Le tableau 9 permet de distinguer certaines tendances.

La taille du réseau social des personnes qui ne vivent plus avec leur conjoint mais ont des enfants survivants est sensiblement inférieure à celle des personnes qui vivent encore en couple; De Jong Gierveld et Dykstra (1997) arrivent à des 
TABLEAU 8 - Caractéristiques sociodémographiques des personnes âgées ne vivant pas avec leur conjoint et ayant des enfants survivants, Québec, $1996^{\text {a }}$

\begin{tabular}{lrrrr}
\hline & Veufs(ves) & $\begin{array}{c}\text { Séparés(ées), } \\
\text { divorcés(ées) }\end{array}$ & Total & $\mathrm{N}$ \\
\hline \% de femmes & 84,8 & 45,7 & 78,8 & 743 \\
\hline Âge moyen (âge) & 76,4 & 69,2 & 75,4 & 743 \\
\hline Taille moyenne du mënage & 1,5 & 1,3 & 1,4 & 743 \\
\hline Mode de vie (\%) & & & & \\
Vit seul(e) & 74,2 & 72,8 & 73,6 & 576 \\
Vit avec enfants & 22,0 & 13,6 & 20,3 & 140 \\
Vit avec d'autres & 3,8 & 13,6 & 6,1 & 26 \\
Total & 100,0 & 100,0 & 100,0 & 742 \\
\hline Revenu personnel (\%) & & & & \\
Moins de 10 000 \$ & 27,4 & 26,6 & 27,0 & 126 \\
10 000-19 999 \$ & 58,1 & 47,7 & 55,5 & 303 \\
20 000-29 999 \$ & 8,7 & - & 10,1 & 43 \\
30 000 \$ et plus & 6,0 & - & 7,1 & 31 \\
Total & 100,0 & 100,0 & 100,0 & 503 \\
\hline État de santé (\%) & & & & \\
Excellent, très bon & 40,3 & 36,9 & 39,7 & 280 \\
Bon & 36,2 & 40,1 & 36,9 & 283 \\
Moyen & 16,5 & 15,6 & 16,4 & 127 \\
Mauvais & 7,0 & - & 7,0 & 60 \\
Total & 100,0 & 100,0 & 100,0 & 750 \\
\hline IES moyen (valeur) & 0,77 & 0,76 & 0,77 & 668 \\
\hline Source & & & & \\
\hline
\end{tabular}

Source : Statistique Canada, Enquête sociale générale, cycle 11, 1996.

a. Les répondants célibataires ont été exclus de ce tableau en raison de leur faible nombre $(n=10)$. Italiques et - : la variabilité d'échantillonnage relative à ces estimations est élevée (trop dans certains cas pour que les estimations soient publiées).

résultats similaires. Cette différence est attribuable, d'une part, au nombre de personnes apparentées, systématiquement moins élevé pour les veufs et les divorcés. Au-delà des différences d'âge, les événements vécus par ceux-ci semblent exercer le mème genre d'impact sur leurs relations familiales. Il est intèressant à cet égard de comparer la situation des femmes âgées vivant avec leur conjoint et celle de la population veuve du tableau 9 (fortement féminine). Les premières se disent proches de 3,6 personnes apparentées en moyenne, la seconde de 2,4. On peut formuler l'hypothèse que certains liens avec la bellefamille tendent à se perdre après un veuvage ou un divorce. 
TABLEAU 9 - Réseau social des personnes âgées ne vivant pas avec leur conjoint et ayant des enfants survivants, Québec, 1996

\begin{tabular}{|c|c|c|c|}
\hline & Veufs(ves) & $\begin{array}{l}\text { Séparés(ées), } \\
\text { divorcés(ées) }\end{array}$ & Total \\
\hline Nombre moyen d'enfants survivants & 3,8 & 3,7 & 3,8 \\
\hline $\begin{array}{l}\text { Nombre moyen de frères et sœurs } \\
\text { survivants }\end{array}$ & 3,3 & 3,4 & 3,3 \\
\hline $\begin{array}{l}\text { Nombre moyen de personnes } \\
\text { apparentées proches }\end{array}$ & 2,4 & 2,6 & 2,4 \\
\hline Nombre moyen d'amis proches & 2,9 & 3,9 & 3,1 \\
\hline Taille moyenne du réseau social & 12,5 & 13,7 & 12,7 \\
\hline
\end{tabular}

Source : Statistique Canada, Enquête sociale générale, cycle 11, 1996.

D'autre part, la différence dans la taille moyenne du réseau social est liée au nombre moyen d'amis proches, plus faible ici, chez les veufs surtout, que chez les personnes vivant en couple (tableau 3). Le nombre d'amis déclaré par les personnes séparées ou divorcées constitue la principale différence entre elles et les personnes veuves en ce qui concerne la composition du réseau social. Même si la population divorcée est plus jeune et plus masculine que la population veuve, cette différence résulte probablement aussi de la dégradation des relations familiales après le divorce : n'ayant jamais pu compter autant sur le réconfort ou le soutien de leur famille, les divorcés les ont cherchés auprès de leurs amis.

Une personne veuve sur quatre recevait des soins en 1996 (tableau 10); la proportion diminue, à $15 \%$, chez les personnes divorcées, de 7 ans plus jeunes en moyenne. Dans la moitié des cas, une seule personne donnait des soins, quel que soit l'état matrimonial des bénéficiaires de cette aide. On remarque que les divorcés ont un réseau de soutien informel plus diversifié que les veufs puisque, dans presque $23 \%$ des cas, ils ont reçu des soins d'au moins trois personnes différentes. C'est très certainement une raison supplémentaire de croire qu'ils ne comptent pas autant que les veufs sur l'aide de leurs enfants et, de façon générale, de leur famille proche.

En moyenne, les aînés ne vivant pas avec leur conjoint et ayant des enfants survivants reçoivent des soins de 1,6 personne, comme les personnes mariées. La taille moyenne du réseau de soutien informel des personnes âgées est donc stable, peu importe leur situation à l'égard d'un conjoint ou la taille de leur réseau social. 
TABLEAU 10 - Réseau de soutien informel des personnes âgées ne vivant pas avec leur conjoint et ayant des enfants survivants, Québec, $1996^{\text {a }}$

\begin{tabular}{lrrr}
\hline & Veufs(ves) & $\begin{array}{c}\text { Séparés(ées), } \\
\text { divorcés(ées) }\end{array}$ & Total \\
\hline \% de personnes âgées recevant des soins & 25,8 & 15,4 & 24,8 \\
IES moyen & 0,61 & 0,60 & 0,61 \\
Taille du ménage & 1,3 & 1,5 & 1,3 \\
\hline Nombre moyen de fournisseurs de soins & & & \\
1 & 53,7 & 52,2 & 54,3 \\
2 & 39,4 & 25,0 & 37,6 \\
3 et plus & - & 22,7 & 8,1 \\
$\quad$ Moyenne & 1,6 & 1,7 & 1,6 \\
\hline Répartition selon que le fournisseur de & & & \\
soins (\%) : & & & \\
$\quad$ Vit dans le même ménage que l'aîné & 23,0 & 27,1 & 24,3 \\
$\quad$ Vit à l'extérieur du ménage de l'aîné & 77,0 & 72,9 & 75,7 \\
$\quad$ Total & 100,0 & 100,0 & 100,0 \\
\hline
\end{tabular}

Source : Statistique Canada, Enquête sociale générale, cycle 11, 1996.

a. Italiques et - : la variabilité d'échantillonnage relative à ces estimations est élevée (trop dans certains cas pour que les estimations soient publiées).

Cette stabilité apparente du réseau de soutien informel des aînés masque cependant une diversité accrue chez ceux qui ne vivent pas avec leur conjoint; ces résultats confirment ceux de Barrett et Lynch (1999). Trois fois sur quatre, les aidants vivent à l'extérieur du ménage de l'aîné, soit une proportion plus élevée que pour la population vivant encore en couple.

Deux sources d'aide paraissent essentielles au maintien de l'autonomie de ces aînés : les enfants (filles et fils) et le réseau formel (tableau 11). On constate des variations selon l'état matrimonial dans l'aide fournie par les enfants : alors que les filles sont le premier fournisseur de soins des personnes veuves, cette place est occupée par le réseau formel pour les divorcés, la fille n'arrivant qu'en troisième lieu, derrière les fils. Cette différence témoigne probablement de la dégradation des relations des parents avec leurs enfants après le divorce, en particulier avec les filles. Chez les personnes veuves, le réseau de soutien formel demeure également très important puisqu'un tiers des soins sont fournis par lui. Cette situation laisse croire que la présence d'enfants ne sera pas plus que la présence d'un conjoint une garantie que la pression sur le réseau formel sera moindre dans l'avenir. Il faut enfin souligner le rôle non négligeable des amis et voisins auprès des personnes divorcées. 
TABLEAU 11 - Foumisseurs de soins des personnes âgées ne vivant pas avec leur conjoint et ayant des enfants survivants, Québec, 1996 (\%) a

\begin{tabular}{lccc}
\hline & Veufs(ves) & $\begin{array}{c}\text { Séparés(ées), } \\
\text { divorcés(ées) }\end{array}$ & Total \\
\hline Fils & 14,1 & 32,4 & 16,1 \\
Filles & 37,1 & 13,8 & 34,4 \\
Membres de la famille ${ }^{b}$ & 10,1 & - & 9,4 \\
Amis et voisins & 4,9 & 10,6 & 6,8 \\
Réseau de soutien formel $c$ & 33,8 & 35,0 & 33,1 \\
Total & 100,0 & 100,0 & 100,0 \\
\hline
\end{tabular}

Source : Statistique Canada, Enquête sociale générale, cycle 11, 1996.

a. Italiques et - : la variabilité d'échantillonnage relative à ces estimations est êlevée (trop dans certains cas pour que les estimations soient publiées).

b. Frères, sœurs, beaux-frères, belles-sœurs, petits-fils, petites-filles, gendres, brus, cousins et cousines.

c. Aide reçue d'un organisme non gouvernemental, d'un employé rémunéré ou d'un organisme gouvernemental.

\section{Les personnes âgées ne vivant pas avec leur conjoint et n'ayant plus d'enfants}

On peut faire l'hypothèse que les personnes âgées ne vivant pas avec leur conjoint et n'ayant plus d'enfants survivants sont celles qui auront le plus de difficulté à obtenir dans leur réseau social les soins dont elles ont besoin pour demeurer dans leur communauté. Ce sont également les plus susceptibles d'avoir recours au réseau de soutien formel et aux établissements de santé, et de souffrir d'isolement et de solitude. Environ une personne âgée sur dix était dans cette situation au Québec en 1996, proportion suffisante pour qu'on étudie cette population de plus près. Toutefois, l'enquête n'a touché qu'un faible nombre de répondants sans conjoint ni enfants, et les résultats présentés sont sujet à une variabilité d'échantillonnage élevée qui limite souvent l'interprëtation.

Le tableau 12 montre qu'il s'agit d'une population à prédominance féminine puisque deux personnes sur trois sont des femmes. L'âge moyen est de 75 ans, avec de légères variations selon le sexe, la population masculine étant un peu plus jeune. C'est pour l'état matrimonial et le mode de vie qu'apparaissent les différences les plus marquées : si, dans l'ensemble, $63 \%$ des individus sont célibataires, la proportion est de $84 \% \mathrm{chez}$ les hommes et de seulement $54 \%$ chez les femmes. Chez ces dernières, près de $36 \%$ sont veuves. Ces différences ont un 
TABLEAU 12 - Caractéristiques socio-démographiques des personnes âgées sans enfant survivant et ne vivant pas avec leur conjoint, Québec, 1996 a

\begin{tabular}{|c|c|c|c|c|}
\hline & Hommes & Femmes & Total & $\mathrm{N}$ \\
\hline Répartition selon le sexe (\%) & 29,1 & 70,9 & 100,0 & 199 \\
\hline Âge moyen (âge) & 72,1 & 76,0 & 74,9 & 199 \\
\hline Taille moyenne du ménage & 2,0 & 1,3 & 1,5 & 199 \\
\hline \multicolumn{5}{|l|}{ Etat matrimonial (\%) } \\
\hline Veufs(ves) & - & 36,2 & 28,2 & 59 \\
\hline Séparés(ées), divorcés(ées) & - & 9,6 & 8,9 & 12 \\
\hline Célibataires & 83,7 & 54,2 & 62,9 & 127 \\
\hline Total & 100,0 & 100,0 & 100,0 & 198 \\
\hline \multicolumn{5}{|l|}{ Mode de vie (\%) } \\
\hline Vit seul(e) & 56,2 & 75,5 & 69,9 & 157 \\
\hline Vit avec d'autres & 43,8 & 24,5 & 30,1 & 42 \\
\hline Total & 100,0 & 100,0 & 100,0 & 199 \\
\hline \multicolumn{5}{|l|}{ Revenu personnel (\%) } \\
\hline Moins de $10000 \$$ & - & 20,1 & 17,8 & 19 \\
\hline $10000-19999 \$$ & 54,1 & 37,7 & 43,1 & 48 \\
\hline $20000-29999 \$$ & - & - & 13,0 & 24 \\
\hline $30000 \$$ et plus & - & 30,7 & 26,2 & 27 \\
\hline Total & 100,0 & 100,0 & 100,0 & 118 \\
\hline \multicolumn{5}{|l|}{ Etat de santé $(\%)$} \\
\hline Excellent, très bon & 42,2 & 45,5 & 44,6 & 89 \\
\hline Bon & 32,9 & 29,8 & 30,8 & 64 \\
\hline Moyen & 22,9 & 21,6 & 21,9 & 35 \\
\hline Mauvais & - & - & - & 8 \\
\hline Total & 100,0 & 100,0 & 100,0 & 196 \\
\hline IES moyen (valeur) & 0,76 & 0,78 & 0,76 & 181 \\
\hline
\end{tabular}

Source : Statistique Canada, Enquête sociale générale, cycle 11, 1996.

a. Italiques et - : la variabilité d'échantillonnage relative à ces estimations est ėlevée (trop dans certains cas pour que les estimations soient publiées).

impact sur le mode de vie: si, globalement, la plus grande partie de cette population vit seule $(70 \%)$, ce n'est le cas que de $56 \%$ des hommes contre $75 \%$ des femmes.

Cette proportion de femmes vivant seules - dont un certain nombre sont veuves - explique probablement la proportion de femmes ayant des revenus égaux ou supérieurs à 30000 dollars (Statistique Canada, 1998), sans doute grâce au cumul de leur revenu de sécurité de la vieillesse et des revenus de réversion ou de retraite de leur conjoint disparu. 
Ces résultats montrent qu'au sein de la population sans conjoint ni enfants survivants, les hommes, peu nombreux, sont célibataires pour la plupart et habitent souvent avec d'autres personnes. Les femmes, beaucoup plus nombreuses, sont pour près de la moitié veuves ou divorcées et vivent le plus souvent seules. Ces différences ont de l'importance pour l'analyse du réseau de soutien informel car elles laissent croire que les hommes, pourtant privés de l'aide potentielle d'un conjoint et d'enfants, sont mieux entourẻs pour recevoir des soins à la vieillesse que les femmes. Le seul avantage de ces dernières réside dans un revenu supérieur susceptible de leur faciliter l'accès aux services formels, notamment à l'assistance d'employés rémunérés. Il faut par conséquent s'attendre à constater d'importantes différences dans le réseau social et le réseau de soutien informel des hommes et des femmes sans conjoint ni enfants.

Le tableau 13 montre que, des trois groupes étudiés dans cet article, les personnes sans conjoint ni enfants sont celles qui ont le réseau social le plus petit : 9 personnes en moyenne. Mais en rapportant ce résultat à ceux du tableau 9, il est facile de voir que les différences s'expliquent, en fait, par l'absence d'enfants, car les nombres moyens de frères et sœurs, de personnes apparentées et d'amis sont tout à fait comparables.

$\mathrm{Au}$ sein de la population sans conjoint et sans enfants, les hommes, encore une fois, ont un réseau social plus étendu, notamment parce qu'ils se reconnaissent un plus grand nombre d'amis. À cet égard, au-delà des différences d'état matrimonial (les personnes célibataires auraient plus d'amis que les autres), il convient de retenir l'hypothèse que les hommes donnent souvent au mot "proche " un sens moins restrictif que les femmes.

Une différence importante entre les sexes existe également en ce qui a trait au nombre moyen de frères et de sœurs, les

TABLEAU 13-Réseau social des personnes âgées sans enfant survivant et ne vivant pas avec leur conjoint, Québec, 1996

\begin{tabular}{lccc}
\hline & Hommes & Femmes & Total \\
\hline Nombre moyen de frères et sœurs & 3,9 & 2,8 & 3,1 \\
Nombre moyen de personnes & 2,2 & 2,8 & 2,6 \\
apparentées proches & & & \\
Nombre moyen d'amis proches & 4,0 & 3,1 & 3,3 \\
Taille moyenne du réseau social & 10,1 & 8,7 & 9,0 \\
\hline
\end{tabular}

Source : Statistique Canada, Enquête sociale générale, cycle 11, 1996. 
TABLEAU 14 - Réseau de soutien informel des personnes âgées sans enfant survivant et ne vivant pas avec leur conjoint, Québec, $1996^{\text {a }}$

\begin{tabular}{lrrr}
\hline & Hommes & Femmes & Total \\
\hline \% de personnes âgées recevant des soins & 21,0 & 23,1 & 22,5 \\
IES moyen & 0,43 & 0,57 & 0,53 \\
Taille du ménage & 2,5 & 1,4 & 1,7 \\
\hline Nombre moyen de fournisseurs de soins & & & \\
1 & - & 76,2 & 63,0 \\
2 & - & 20,0 & 19,0 \\
3 et plus & 56,1 & - & 18,0 \\
$\quad$ Moyenne & 2,7 & 1,3 & 1,7 \\
\hline Répartition (\%) selon que le fournisseur & & & \\
de soins : & - & - & 19,8 \\
$\quad$ Vit dans le même ménage que l'ainé & 66,7 & 91,1 & 80,2 \\
$\quad$ Vit à l'extérieur du ménage de l'aìné & 100,0 & 100,0 & 100,0 \\
$\quad$ Total &
\end{tabular}

Source : Statistique Canada, Enquête sociale générale, cycle 11, 1996.

a. Italiques et - : la variabilité d'échantillonnage relative à ces estimations est élevée (trop dans certains cas pour que les estimations soient publiées).

hommes étant plus favorisés. L'âge moyen des deux populations ne peut certainement pas expliquer en totalité cet écart, et on peut supposer qu'il a un lien avec l'espérance de vie supérieure des femmes. La probabilité qu'un homme perde une sœur est inférieure à celle qu'une femme perde un frère. Les hommes seraient ainsi avantagés au plan de la fratrie aux âges avancés.

Il n'est pas exclu que ces résultats soient également liés à la proportion de célibataires (tableau 12). Au sein des cohortes étudiées, un nombre important de gens sont probablement demeurés célibataires afin de servir l'Église. Pour les hommes, la tradition a pu faire en sorte que cette situation se produise plus fréquemment au sein de familles nombreuses que pour les femmes, pour des raisons d'utilité sociale des enfants.

Un peu plus d'une personne sur cinq sans conjoint et sans enfants recevait des soins en 1996 (tableau 14). Le nombre moyen de fournisseurs de soins était de 1,7 , soit un nombre équivalent à ceux qu'on trouve aux tableaux 4 et 10 . Ce résultat a de quoi surprendre, mais le nombre varie notablement selon le sexe, ce qui n'était pas le cas au tableau 4 ou (selon l'état matrimonial) au tableau 10. La taille du réseau de soutien informel est beaucoup plus élevée pour les hommes sans conjoint et sans enfants que pour les femmes: presque 3 , comparativement à 1,3 . Un peu plus de $56 \%$ des hommes ont 
déclaré recevoir des soins d'au moins trois personnes différentes, mais moins de $5 \%$ des femmes ont fait de même (données non présentées). Trois femmes sur quatre n'ont qu'un aidant. De plus, leurs aidants vivent presque toujours à l'extérieur du ménage; au contraire, les hommes reçoivent le tiers de leurs soins d'aidants vivant avec eux.

Les femmes, encore une fois, paraissent désavantagées ou, du moins, plus susceptibles de devoir compter sur l'assistance du réseau formel. Tout indique que les hommes, en particulier les célibataires, ont pris les moyens, en allant fréquemment vivre avec d'autres personnes, de recevoir l'aide et les soins dont ils pourraient avoir besoin dans leur vieillesse.

Les rêsultats présentés ici laissent également croire que la taille du réseau social n'est pas nécessairement associée à celle du réseau de soutien informel. Les hommes qui vivent sans conjoint et n'ont pas d'enfants ont un réseau social relativement limité (10 personnes) mais, en moyenne, reçoivent des soins de presque trois aidants différents, dont les deux tiers vivent à l'extérieur de leur ménage. À l'inverse, les hommes vivant en couple ont un réseau social étendu mais ne reçoivent des soins, en moyenne, que de 1,6 personne. Ces résultats peuvent apparaitre comme un appui à la théorie " hiérarchique compensatoire " formulée par Shanas (1979) et Cantor (1979), selon laquelle les ainés sollicitent d'abord leurs proches afin de recevoir des soins.

Les différences marquées du tableau 14 se reflètent au tableau 15, notamment en ce qui a trait au recours au réseau de soutien formel. Dans l'ensemble, la moitié des dyades de

TABLEAU 15 - Fournisseurs de soins des personnes âgées sans enfant survivant et ne vivant pas avec leur conjoint, Québec, 1996 (\%) a

\begin{tabular}{|c|c|c|c|}
\hline & Hommes & Femmes & Total \\
\hline Famille $^{b}$ & 58,9 & 23,6 & 38,6 \\
\hline Amis et voisins & - & - & - \\
\hline Réseau de soutien formel c & 30,7 & 66,5 & 51,2 \\
\hline Total & 100,0 & 100,0 & 100,0 \\
\hline
\end{tabular}

Source : Statistique Canada, Enquête sociale générale, cycle 11, 1996.

a. Italiques et - : la variabilité d'échantillonnage relative à ces estimations est élevée (trop dans certains cas pour que les estimations soient publiées).

b. Frères, scurs, beaux-frères, belles-sours, petites-filles, neveux, nièces, cousins et cousines.

c. Aide reçue d'un organisme non gouvernemental, d'un employé rémunéré ou d'un organisme gouvernemental. 
soins impliquent ce réseau, mais la proportion atteint les deux tiers pour les femmes. La famille, notamment les frères, soeurs, beaux-frères et belles-sours, est la principale source de soins des hommes. On peut penser que, dans bien des cas, ces acteurs du réseau de soutien informel vivent dans le même ménage que l'homme âgé célibataire dont ils s'occupent. La famille joue un rôle beaucoup moins important pour les femmes, bien qu'elles soient assez souvent veuves.

\section{SYNTHESE ET CONCLUSION}

Notre but était de décrire la taille et la composition du réseau de soutien informel des personnes âgées en tenant compte de la présence du conjoint et des enfants, afin de mieux comprendre les stratégies utilisées par les aînés pour conserver leur autonomie et demeurer dans leur communauté.

L'analyse fait ressortir des différences marquées entre les trois groupes étudiés: les personnes âgées qui vivent sans conjoint (avec ou sans enfants) sont plus âgées, sont en moins bonne santé et conséquemment ont besoin de plus de soins que celles qui vivent encore avec leur conjoint. S'il y avait une hièrarchie à ètablir entre les personnes âgées afin de désigner les mieux nanties en termes de soutien social, les plus avantagés seraient sans nul doute les hommes vivant avec leur conjointe. Ces derniers disposent en effet du réseau social le plus étendu et reçoivent la plus grande part des soins dont ils ont besoin d'aidants qui vivent dans le même ménage qu'eux, géné-ralement leur conjointe. Ce sont également ceux qui font appel le moins souvent à l'assistance du réseau formel.

Les aînés les moins avantagés sont certainement les femmes sans conjoint ni enfants survivants. Plus âgées, et donc en moins bonne santé, elles ont un réseau social et un réseau de soutien informel très limités, et la presque-totalité des soins qu'elles reçoivent provient d'aidants extérieurs à leur ménage, le plus souvent du réseau formel. Vivant de surcroît souvent seules, elles semblent cependant moins exposées à la précaritê financière; leur aisance relative pourrait leur permettre de se procurer plus facilement auprès du réseau formel une partie de l'assistance dont elles ont besoin.

Nous devons signaler les limites de l'analyse présentée ici, liees à l'insuffisance du nombre de cas. L'utilisation des techniques statistiques de régression multivariee permettra de mieux 
mettre en évidence, dans un prochain article, les liens entre l'utilisation des services formels et (ou) informels et les caractéristiques sociodémographiques des personnes âgées.

Selon les théories répandues en gërontologie, plus les ainés sont isolés de leur conjoint et de leurs enfants, plus ils font appel aux services formels de soins. Les résultats de cette étude montrent que le rôle du réseau de soutien formel, constitué des organismes non gouvernementaux, des employés rémunérés et du gouvernement, demeure essentiel au maintien à domicile des personnes âgées, en particulier pour les femmes sans conjoint ni enfants, les personnes séparées ou divorcées ayant des enfants survivants et, de façon inattendue, les femmes âgées vivant encore avec leur conjoint. Dans toutes ces situations, ce réseau est le principal fournisseur de soins des personnes âgées.

Les résultats présentés dans cet article permettent non seulement d'évaluer le réseau de soutien informel des Québécois âgés d'aujourd'hui, mais également d'alimenter la réflexion sur l'avenir. L'arrivée des générations du baby-boom à 65 ans dès 2011 devrait produire un accroissement du nombre de personnes âgées sans conjoint et (ou) sans enfants (Connidis et McMullin, 1994; Grundy, 1995; Gee, 1995). D'une cohorte à l'autre comme d'un individu à l'autre, la taille, la composition et la disponibilité du réseau social évoluent, notamment en raison des comportements démographiques adoptés durant le cycle de vie. Or, les baby-boomers auront, plus que quiconque auparavant, compromis, du moins théoriquement, le soutien qu'ils auraient pu espérer recevoir de leur conjoint et de leurs enfants une fois entrés dans la vieillesse, puisqu'ils sont en bonne partie responsables de la hausse du nombre de divorces ainsi que de la baisse spectaculaire de la fécondité au Québec. Il ne fait pas de doute que le réseau formel de maintien à domicile sera appelé à demeurer un acteur de premier plan pour les personnes âgées et que la demande de ce genre de services ira croissant.

Il faut, en terminant, souligner l'importance de se doter, au Québec comme au Canada, de bases de données étendues permettant l'étude des phénomènes et situations reliés au vieillissement. Jusqu'ici, très peu d'enquètes nationales ont porté exclusivement sur la population âgée, contrairement à la pratique américaine par exemple. L'efficacité des futures politiques publiques de maintien à domicile repose sur une analyse détaillée et fiable des besoins en matière de santé fonctionnelle et de 
l'utilisation actuelle des systèmes en place. C'est une réalité que les enquêtes spécifiques au vieillissement doivent s'efforcer de mieux saisir; l'Enquête sociale générale que mènera Statistique Canada en 2002 sur la population âgée de 45 ans et plus permettra sans doute de combler davantage ces besoins.

\section{RÉFÉRENCES BIBLIOGRAPHIQUES}

ANTONUCCI, T. C., et H. AKIYAMA. 1987. "Social networks in adult life and a preliminary examination of the convoy model ", Journal of Gerontology, 42, $5:$ 519-527.

BARRETT, A. E., et S. C. LYNCH. 1999. "Caregiving networks of elderly persons: Variation by marital status ", The Gerontologist, 39, $6: 695-704$.

BEGGS, J. J., V. A. HAINES et J. S. HURLBERT. 1996. "Situational contingencies surrounding the receipt of informal support ", Social Force, $75: 201-222$.

BERTHELOT, J.-M., R. ROBERGE et M. C. WOLSON. 1993. "Le calcul de l'espérance de vie ajustée sur la santé pour l'Ontario ", Colloque INSERM, Montpellier, France, John Libbey Eurotext Ltd. : 161-172.

CAFFERATA, G. L. 1987. "Marital status, living arrangements, and the use of health services by elderly persons ", Joumal of Gerontology, 42:613-618.

CAMPBELL, L. D., I. A. CONNIDIS et L. DAVIES. 1999. "Sibling ties in later life ", Joumal of Family Issues, 20, $1: 114-148$.

CANTOR, M. H. 1979. "Neighbors and friends. An overlooked resource in the informal support system ", Research on Aging, $1: 434-463$.

CANTOR, M. H. 1994. "Family caregiving: Social care", dans M. CANTOR, éd. Family Caregiving: Agenda for the Future. San Francisco, CA, American Society on Aging : 1-19.

CHAPMAN, N. J. 1989. "Gender, marital status and childlessness of older persons and the availability of informal assistance ", dans M. D. PETERSEN et D. L. WHITE, éd. Health Care of the Elderly: An Information Source Book. Newbury Park, Sage : 277-328.

CHAPPELL, Neena L. 1991. "Living arrangements and sources of caregiving ", Journal of Gerontology: Social Sciences, 46, 1 : S1-8.

CONNIDIS, I. A., et J. A. MCMULLIN. 1994. "Social support in older ages: Assessing the impact of marital and parent status ", La Revue canadienne du vieillissement/Canadian Journal on Aging, 13 : 510527.

DE JONG GIERVELD, J., et P. A. DYKSTRA. 1997. "The long-term consequences of divorce for fathers", dans International Union for the Scientific Study of Population, International Population Conference, Beijing, 1997, 2 : 849-866. 
DYSTRA, P. A. 1997. "The consequences of divorce for intergenerational exchange within the family ", Bevolking en Gezin (Bruxelles, Belgique), $1: 75-94$.

FURSTENBERG, F. F., S. D. HOFFMAN et L. SHRESTHA. 1995. "The effect of divorce on intergenerational transfers: New evidence ", Demography, $32: 319-333$.

GEE, Ellen M. 1995 "Situation familiale des personnes d'âge mûr ", dans R. BEAUJOT, éd. La Famille au long de la vie. Statistique Canada, no 91-543F au catalogue : 83-122.

GIRANDA, Melanie, James E. LUK et Kathryn A. ATCHISON. 1999. "Social networks of elders without children ", Journal of Gerontological Social Work, 31, 1/2:63-84.

GRUNDY, Emily. 1995. "Demographic influences on the future of family care "dans I. ALLEN et E. PERKINS, éd. The Future of Family Care for Older People. Londres, HMSO.

KEATING, Norah, Janet FAST, Judith FREDERICK, Kelly CRANSWICK et Cathryn PERRIER. 1999. Soins aux personnes âgees au Canada: contexte, contenu et conséquences. Statistique Canada, no 89-570 au catalogue, $134 \mathrm{p}$.

KEITH, P. M. 1985. "Financial well-being of older divorced/separated men and women: Findings from a panel study ", Journal of Divorce, $9,1: 61-72$.

LITWAK, E. 1985. Helping the Elderly: The Complementary Roles of Informal Networks and Formal Systems. New York, The Guilford Press.

MARTEL, Laurent. 1995. L'Aide reçue et l'aide foumie par les personnes âgées du Québec. Université de Montréal, Département de démographie, mémoire de maitrise (sous la direction de Jacques Lègarè), $85 \mathrm{p}$.

MARTEL, Laurent. 1998. "Quel isolement durant la vieillesse pour les femmes à l'origine du baby-boom ", dans AIDELF. Morbidité, mortalité : problèmes de mesure, facteurs d'évolution, essai de prospective. PUF : 639-653.

MARTEL, Laurent, et Yves CARRIËRE. 2001. "Descriptive analysis of the effects of marital dissolution on living arrangements of older persons: An international comparison ", soumis à Genus.

MORGAN, D. L., et S. J. MARCH. 1992. "The impact of life events on networks of personal relationships: A comparison of widowhood and caring for a spouse with Alzheimer's disease ", Journal of Social and Personal Relationships, 9 : 563-584.

NAULT, F. 1997. "Diminution des écarts de mortalité, de 1978 à 1995 ", Rapports sur la santé, Statistique Canada, no 82-003 au catalogue, 9, $1: 37-43$.

PELLETIER, Louis. 1992. "Vieillir en institution ou à domicile ? Les facteurs associès à l'hébergement des personnes âgées ", Espace, populations, sociétés, $1: 71-86$. 
PEZZIN, Liliana E., et Barbara STEINBERG SCHONE. 1999. "Parental marital disruption and intergenerational transfers: An analysis of lone elderly parents and their children ", Demography, 36, 3 : 287297.

SHANAS, E., J. FRIES, H. P. MILHOJ et J. STEINHOWER. 1968. Old People in Three Industrial Societies. New York et Londres : Atherton and Routledge and Kegan Paul, 478 p.

SHANAS, Ethel. 1979. "The family as a social support system in old age ", The Gerontologist, 19, 2 : 169-174.

STATISTIQUE CANADA. 1998. "Effets de l'entourage des personnes âgées sur leur condition socio-économique ", dans Rapport sur l'état de la population du Canada 1997. Statistique Canada, no 91-209 au catalogue : 135-190.

STONE, Leroy O., et Carolyn ROSENTHAL. 1997. "The profile of the social networks of Canada's elderly: An analysis of 1990 General Social Survey data ", dans H. LITWIN, ed. The Social Networks of Older People. A Cross-national Analysis. Londres, Praeger : 78-97.

STRAIN, L. A., et B. J. PAYNE. 1992. "Social networks and patterns of social interaction among ever-single and separated/divorced elderly Canadians ", Canadian Journal on Aging/La Reiue canadienne du vieillissement, 11, 1: 31-53.

STOLLER, E. P., et K. L. PUGLIESI. 1991. "Size and effectiveness of informal helping networks: A panel study of older people in the community ", Journal of Health and Social Behavior, 32 : 180-191.

THORNTON, M. C., et H. K. WHITE-MEANS. 1993. "Socio-demographic correlates of the size and composition of informal caregiver networks among frail ethnic elderly", Journal of Comparative Family Studies, 24 : 235-250.

TROTTIER, Helen, Laurent MARTEL, Christian HOULE, Jean-Marie BERTHELOT et Jacques LÉGARÉ. 2000. "Vieillir chez soi ou en établissement : à quoi cela tient-il ?", Rapports sur la santé, Statistique Canada, no 82-003 au catalogue, 11, 4 : 55-69.

UHLENBERG, P. 1994. "Implications of being divorced in later life ", dans Ageing and the Family: Proceedings of the United Nations International Conference on Ageing Population in the Context of the Family, Kitakyushu (Japan). New York, United Nations : 121-127.

WOLF, Douglas A. 1994. "The elderly and their kin: Patterns of availability and access ", dans L. G. MARTIN et S. H. PRESTON, éd. Demography of Aging. Washington, D. C., National Academy Press: 146-194.

WU, Z., et M. S. POLLARD, 1998. "Social support among unmarried childless eiderly persons ", Journal of Gerontology: Social Sciences, 53B, 6 : S324-S335. 


\begin{abstract}
Laurent MARTEL and Jacques LÉGARÉ

WITH OR WITHOUT THE SUPPORT OF CLOSE FAMILY MEMBERS IN OLD AGE: A DESCRIPTION OF THE ELDERLY'S INFORMAL SUPPORT NETWORKS BASED ON THE PRESENCE OR ABSENCE OF A SPOUSE AND/OR CHILDREN

In a context increasingly marked by the deinstitutionalization of the elderly and tighter control over health care spending, our societies are calling upon greater support from the social networks of individuals experiencing a loss of autonomy. However, these social networks are changing. Before looking at the future availability and structure of these networks, it is worthwhile examining under what circumstances and how the elderly currently use their formal and informal support networks. As home care policies vary from one Canadian province to another, and as the Institut de la statistique du Québec (ISQ) had funded interviews of an oversample of 700 elderly persons in the General Social Survey (Statistics Canada, 1996), the present analysis focuses on the situation of Quebec seniors receiving care due to long-term health problems. Three specific situations are examined: the situation of seniors still living with their spouse, that of seniors no longer living with their spouse but who still have surviving children, and that of seniors presumably on their own, i.e. no longer living with their spouse and without surviving children. Clearly, not all seniors require health care, but the findings do raise a number of questions for the future since the availability of informal networks will be markedly reduced.
\end{abstract}

